\title{
Finite-Time Stability Analysis of Switched Genetic Regulatory Networks
}

\author{
Lizi Yin ${ }^{1,2}$ \\ ${ }^{1}$ School of Control Science and Engineering, Shandong University, Jinan 250061, China \\ ${ }^{2}$ School of Mathematical Sciences, University of Jinan, Jinan 250022, China \\ Correspondence should be addressed to Lizi Yin; ss_yinlz@ujn.edu.cn
}

Received 11 October 2013; Revised 15 December 2013; Accepted 15 December 2013; Published 19 January 2014

Academic Editor: Qiankun Song

Copyright (C) 2014 Lizi Yin. This is an open access article distributed under the Creative Commons Attribution License, which permits unrestricted use, distribution, and reproduction in any medium, provided the original work is properly cited.

\begin{abstract}
This paper investigates the finite-time stability problem of switching genetic regulatory networks (GRNs) with interval time-varying delays and unbounded continuous distributed delays. Based on the piecewise Lyapunov-Krasovskii functional and the average dwell time method, some new finite-time stability criteria are obtained in the form of linear matrix inequalities (LMIs), which are easy to be confirmed by the Matlab toolbox. The finite-time stability is taken into account in switching genetic regulatory networks for the first time and the average dwell time of the switching signal is obtained. Two numerical examples are presented to illustrate the effectiveness of our results.
\end{abstract}

\section{Introduction}

In the last decade or so, the genetic regulatory networks (GRNs) have become an important research area in molecular Biology. On the one hand, how to construct GRNs from gene expression data; on the other hand, what is the dynamic characteristics of gene regulatory networks. The stability is one of vital dynamic characteristics of GRNs and is researched in this paper. There are also many references on the stability of GRNs [1-6].

High throughput biological experiments have proved that time delays were ubiquitous in GRNs. The existence of time delays influences the stability of GRNs, which can give rise to oscillatory or unstable networks. Therefore, it is necessary to study the influences of time delays for the stability of GRNs. There are a few theoretical results on GRNs with time delays [7-15]. In [2], random time delays are taken into account, and some stability criteria for the uncertain delayed genetic networks with SUM regulatory logic where each transcription factor acts additively to regulate a gene were obtained. In [12], some stochastic asymptotic stability conditions were established for a class of uncertain stochastic genetic regulatory networks with mixed time-varying delays by constructing appropriate Lyapunov-Krasovskii functionals and employing stochastic analysis method. In [13, 14], authors studied GRNs with constant delay, and asymptotical stability criteria were proposed for GRNs with interval timevarying delays and nonlinear disturbance in [10].

Cell-cycle regulatory processes can be viewed as finitestate processes. So some complex GRNs were described by continuous time switched system. Usually, a finite state Markov chain was used to simulate this switch process [1619]. In [17], authors investigate the global robust stability of uncertain stochastic GRNs with Markovian switching process. In [19], control theory and mathematical tools were used to analyze passivity for the stochastic Markovian switching GRNs with time-varying delays. Some other methods are also used to prove switched GRNs' stability. In the literature [20], authors used an average dwell time approach to consider exponential stability of switched GRNs with time delays.

Both procedures of these proteins regulate gene expression and gene translated protein that are sometimes accomplished in a relatively short period of time. So it is realistically significant to research the finite-time stability of GRNs and some articles have researched the finite-time stability $[21,22]$. There are few references about the finite-time stability of GRNs; we research only the literature [23]. In [23], the authors considered finite-time robust stability of uncertain stochastic reaction-diffusion GRNs with time delays. The finite-time stability of GRNs is the main contents in our paper. 
Motivated by the above discussions, we analyze finitetime stability of switching genetic regulatory networks with interval time-varying delays and continuous distributed delays. Using a novel piecewise Lyapunov-Krasovskii functional and finite-time stable definite, some new finite-time stability criteria are obtained for the switched GRNs. The features of this paper can be summarized as follows. (1) the finite-time stability is researched in the switched GRNs firstly; (2) continuous distributed delays are concerned; (3) all sufficient conditions obtained depend on the time delays.

This paper is organized as follows. In Section 2, model description, some assumptions, definitions, and lemmas are given. In Section 3, some conditions are obtained to ensure the finite-time stability of switched GRNs with interval time-varying delays and continuous distributed delays. Two numerical examples are given to demonstrate the effectiveness of our analysis in Section 4. Finally, conclusions are drawn in Section 5.

Notations. Throughout this paper, $\mathbf{R}, \mathbf{R}^{n}$, and $\mathbf{R}^{n \times m}$ denote, respectively, the set of all real numbers, real $n$-dimensional space, and real $n \times m$-dimensional space. $\mathbf{Z}_{+}$denote the set of all positive integers. $\|\cdot\|$ denote the Euclidean norms in $\mathbf{R}^{n}$. For a vector or matrix $A, A^{T}$ denotes its transpose. For a square matrix $A, \lambda_{\max }(A)$ and $\lambda_{\min }(A)$ denote the maximum eigenvalue and minimum eigenvalue of matrix $A$, respectively, and sym $(A)$ is used to represent $A+A^{T}$. For simplicity, in symmetric block matrices, we often use $*$ to represent the term that is induced by symmetry.

\section{Problem Formulation and Some Preliminaries}

We consider the following genetic regulatory networks:

$$
\begin{gathered}
\dot{m}(t)=-A m(t)+B f\left(p\left(t-\tau_{1}(t)\right)\right)+I, \\
\dot{p}(t)=-C P(t)+D m\left(t-\tau_{2}(t)\right),
\end{gathered}
$$

where $m(t)=\left[m_{1}(t), \ldots, m_{n}(t)\right]^{T}$ is the concentrations of mRNAs, $p(t)=\left[p_{1}(t), \ldots, p_{n}(t)\right]^{T}$ is the concentrations of proteins, $f(\cdot)=\left[f_{1}(\cdot), \ldots, f_{n}(\cdot)\right]^{T}$ is the regulatory functions of mRNAs, $A=\operatorname{diag}\left(a_{1}, \ldots, a_{n}\right)$ is the degradation rates of mRNAs, $C=\operatorname{diag}\left(c_{1}, \ldots, c_{n}\right)$ is the degradation rates of proteins, $D=\operatorname{diag}\left(d_{1}, \ldots, d_{n}\right)$ is the translation rates of proteins, $I=\left[I_{1}, \ldots, I_{n}\right]^{T}$ is the basal rate, and $\tau_{1}(t)$ and $\tau_{2}(t)$ are time-varying delays.

For obtaining our conclusions, we make the following assumptions.

Assumption 1. $f_{i}: \mathbf{R} \rightarrow \mathbf{R}, i=1, \ldots, n$ are monotonically increasing functions with saturation and satisfy

$$
0 \leq \frac{f_{i}(a)-f_{i}(b)}{a-b} \leq u_{i}, \quad \forall a, b \in \mathbf{R}, i=1, \ldots, n,
$$

where $u_{i}, i=1, \ldots, n$ are nonnegative constants.
Assumption 2. $\tau_{1}(t)$ and $\tau_{2}(t)$ are time-varying delays satisfying

$$
\begin{array}{ll}
0 \leq \tau_{11} \leq \tau_{1}(t) \leq \tau_{12}, & \dot{\tau}_{1}(t) \leq \tau_{13}<\infty \\
0 \leq \tau_{21} \leq \tau_{2}(t) \leq \tau_{22}, & \dot{\tau}_{2}(t) \leq \tau_{23}<\infty .
\end{array}
$$

Vectors $m^{*}, p^{*}$ are an equilibrium point of the system (1). Let $x(t)=m(t)-m^{*}, y(t)=p(t)-p^{*}$; we get

$$
\begin{gathered}
\dot{x}(t)=-A x(t)+B g\left(y\left(t-\tau_{1}(t)\right)\right), \\
\dot{y}(t)=-C y(t)+D x\left(t-\tau_{2}(t)\right),
\end{gathered}
$$

where $x(t)=\left[x_{1}(t), \ldots, x_{n}(t)\right]^{T}, y(t)=\left[y_{1}(t), \ldots, y_{n}(t)\right]^{T}$, $g(\cdot)=[g(\cdot), \ldots, g(\cdot)]^{T}$, and $g_{i}\left(y_{i}(t)\right)=f_{i}\left(y_{i}(t)+p_{i}^{*}\right)-f_{i}\left(p_{i}^{*}\right)$.

According to Assumption 1 and the definition of $g_{i}(\cdot)$, we know that $g_{i}(\cdot)$ is bounded; that is, $\exists G>0$, such that $\left|g_{i}(\cdot)\right| \leq$ $G, i=1, \ldots, n$ and satisfies the following sector condition:

$$
0 \leq \frac{g_{i}(a)}{a} \leq u_{i}, \quad \forall a \in \mathbf{R} /\{0\}, i=1, \ldots, n
$$

Let $U=\operatorname{diag}\left(u_{1}, \ldots, u_{n}\right)$.

Sometimes, GRNs were described by continuous time switched system, as in $[17,24,25]$, so system (4) can be described as switching system with switching signal:

$$
\begin{gathered}
\dot{x}(t)=-A_{\sigma(t)} x(t)+B_{\sigma(t)} g\left(y\left(t-\tau_{1}(t)\right)\right), \\
\dot{y}(t)=-C_{\sigma(t)} y(t)+D_{\sigma(t)} x\left(t-\tau_{2}(t)\right),
\end{gathered}
$$

where $\sigma(t):[0, \infty) \rightarrow \mathbf{N}=\{1, \ldots, N\}$ is the switching signal, which is a piecewise constant function depending on time $t$. For each $i \in \mathbf{N}$, the matrices $A_{i}, B_{i}, C_{i}$, and $D_{i}$ are the $i$ th subsystem matrices that are constant matrices of appropriate dimensions.

For the switching signal $\sigma(t)$, we have the following switching sequence:

$$
\left\{\left(i_{0}, t_{0}\right), \ldots,\left(i_{k}, t_{k}\right), \ldots \mid i_{k} \in \mathbf{N}, k=0,1, \ldots\right\},
$$

in other words, when $t \in\left[t_{k}, t_{k+1}\right), i_{k}$ th subsystem is activated.

The initial condition of system (6) is assumed to be

$$
\begin{gathered}
x(t)=\varphi(t), \quad y(t)=\psi(t), \\
-\rho \leq t \leq 0, \quad \rho=\max \left\{\tau_{12}, \tau_{22}\right\} .
\end{gathered}
$$

For proving the theorem, we recall the following definition and lemmas.

Definition 3. The system (6) is said to be finite-time stable with respect to positive real numbers $\left(c_{1}, c_{2}, T\right)$, if

$$
\begin{array}{r}
\|\phi(t)\|_{C^{1}}^{2}+\|\varphi(t)\|_{C^{1}}^{2} \leq c_{1} \Longrightarrow\|x(t)\|^{2}+\|y(t)\|^{2} \leq c_{2}, \\
\forall t \in[0, T],
\end{array}
$$

where $\|\phi(t)\|_{\mathcal{c}^{1}}=\sup _{-\rho \leq t \leq 0}\{\|\phi(t)\|,\|\dot{\phi}(t)\|\},\|\varphi(t)\|_{c^{1}}=$ $\sup _{-\rho \leq t \leq 0}\{\|\varphi(t)\|,\|\dot{\varphi}(t)\|\}$. 
Remark 4. The Lyapunov stability implies that, by starting sufficiently close to the equilibrium point, trajectories can be guaranteed to stay within any specified ball centered at the equilibrium point. It depicts character of the equilibrium point of the system. The finite-time stability implies that, by starting from any specified compact set, trajectories can be guaranteed to stay within a large enough compact set. It depicts character of all solutions of the system and is called the finite-time boundedness in some literatures [21, 22]. In addition, the Lyapunov stability is considered in infinite interval, but the finite-time stability is considered in finite interval.

Remark 5. In [23], the authors considered the finite-time robust stability of GRNs, but in our paper we considered the finite-time stability of switching GRNs, which are more realistic GRNs than that of the [23]. For the first time switched GRNs' finite-time stability is researched.

Definition 6. For any $T \geq t \geq 0$, let $N_{\sigma}(t, T)$ denote the number of switching of $\sigma(t)$ over $(t, T)$. If

$$
N_{\sigma}(t, T) \leq N_{0}+\frac{T-t}{\tau_{a}}
$$

holds for $N_{0} \geq 0, \tau_{a}>0$, then $\tau_{a}>0$ is called the average dwell time and $N_{0}$ is the chatter bound. As commonly used in the literature, we choose $N_{0}=0$.

Lemma 7 (see [26]). For any positive definite matrix $M \in$ $\mathbf{R}^{n \times n}$, there exist a scalar $q>0$ and a vector-valued function $\omega:[0, q] \rightarrow \mathbf{R}^{n}$ such that

$$
\left(\int_{0}^{q} \omega(s) d s\right)^{T} M\left(\int_{0}^{q} \omega(s) d s\right) \leq q \int_{0}^{q} \omega^{T}(s) M \omega(s) d s .
$$

\section{Main Results}

In this section, we present a finite time stability theorem for switching genetic regulatory networks with interval timevarying delays (6).

Theorem 8. For switching system (6) with Assumptions 1 and 2 , given a scalar $\alpha>0$, if there exist symmetric positive definite matrices $P_{1 i}, P_{2 i}, Q_{1 i}, R_{1 i}, R_{2 i}, R_{3 i}, R_{4 i}$, for all $i \in \mathbf{N}$, the diagonal matrix $\Lambda_{m}=\operatorname{diag}\left(\lambda_{m 1}, \ldots, \lambda_{m n}\right) \geq 0, m=1,2$, matrices $Q_{2 i}$, $Q_{3 i}, M_{1 i}, i \in N$, and positive scalars $\mu \geq 1$ such that the following inequalities

$$
\begin{gathered}
{\left[\begin{array}{cc}
Q_{2 i} & M_{1 i} \\
* & Q_{3 i}
\end{array}\right] \geq 0, \quad P_{k i} \leq \mu P_{k j}, \quad k=1,2,} \\
{\left[\begin{array}{cc}
Q_{2 i} & M_{1 i} \\
* & Q_{3 i}
\end{array}\right] \leq \mu\left[\begin{array}{cc}
Q_{2 j} & M_{1 j} \\
* & Q_{3 j}
\end{array}\right], \quad R_{l i} \leq \mu R_{l j},} \\
l=1, \ldots, 4, \forall i, j \in \mathbf{N},
\end{gathered}
$$

$\Xi=\Omega$

$$
\begin{aligned}
+\operatorname{sym} & \left\{L_{1}^{T} R_{1 i} L_{2}+L_{1}^{T}\right. \\
& \times\left[P_{1 i} B_{i}-\tau_{21}^{2} B_{i}^{T} R_{1 i} A_{i}-\left(\tau_{22}-\tau_{21}\right)^{2} B_{i}^{T} R_{2 i} A_{i}\right] \\
& \times L_{10}+L_{2}^{T} R_{2 i} L_{3}+L_{3}^{T} R_{2 i} L_{4}+L_{3}^{T} \\
& \times\left[P_{2 i} D_{i}-\tau_{11}^{2} D_{i}^{T} R_{3 i} C_{i}-\left(\tau_{12}-\tau_{11}\right)^{2} D_{i}^{T} R_{4 i} C_{i}\right] \\
& \times L_{5}+L_{5}^{T} R_{3 i} L_{6}+L_{5}^{T}\left(M_{1 i}+\Lambda_{1} U\right) L_{9}+L_{6}^{T} R_{4 i} L_{7} \\
& \left.+L_{7}^{T} R_{4 i} L_{8}+L_{7}^{T}\left[M_{1 i}\left(1-\tau_{13}\right)+\Lambda_{2} U\right] L_{10}\right\}
\end{aligned}
$$$$
<0, \quad \forall i \in \mathbf{N},
$$

hold, and the average dwell time of the switching signal $\sigma(t)$ satisfies

$$
\tau_{a}>\tau_{a}^{*}=\frac{T \ln \mu}{\ln c_{2} \lambda_{2}-\ln c_{1} \lambda_{1}-\alpha T}
$$

where $\Omega=\operatorname{diag}\left(-2 P_{1 i} A_{i}+Q_{1 i}+\tau_{21}^{2} A_{i}^{T} R_{1 i} A_{i}+\left(\tau_{22}-\tau_{21}\right)^{2}\right.$ $A_{i}^{T} R_{2 i} A_{i}-R_{1 i}-\alpha P_{1 i},-R_{1 i}-R_{2 i},-Q_{1 i}\left(1-\tau_{23}\right)+\tau_{11}^{2} D_{i}^{T}$ $R_{3 i} D_{i}+\left(\tau_{12}-\tau_{11}\right)^{2} D_{i}^{T} R_{4 i} D_{i}-2 R_{2 i},-R_{2 i},-2 P_{2 i} C_{i}+Q_{2 i}+\tau_{11}^{2} C_{i}^{T}$ $R_{3 i} C_{i}+\left(\tau_{12}-\tau_{11}\right)^{2} C_{i}^{T} R_{4 i} C_{i}-R_{3 i}-\alpha P_{2 i},-R_{3 i}-R_{4 i},-Q_{2 i}(1-$ $\left.\tau_{13}\right)-2 R_{4 i},-R_{4 i}, Q_{3 i}-2 \Lambda_{1},-Q_{3 i}\left(1-\tau_{13}\right)+\tau_{21}^{2} B_{i}^{T} R_{1 i} B_{i}+$ $\left.\left(\tau_{22}-\tau_{21}\right)^{2} B_{i}^{T} R_{2 i} B_{i}-2 \Lambda_{2}\right), L_{j}=\left[0_{n \times(j-1) n}, I_{n \times n}, 0_{n \times(11-j) n}\right], j=$ $1, \ldots, 10, \lambda_{1}=\max _{i \in \mathbf{N}}\left\{\lambda_{\text {max }}\left(P_{1 i}\right)+\tau_{22} \lambda_{\text {max }}\left(Q_{1 i}\right)+\lambda_{\text {max }}\right.$ $\left(P_{2 i}\right)+\tau_{12} \lambda_{\max }\left(Q_{2 i}\right)+\tau_{12} \lambda_{\max }\left(Q_{3 i}\right) \lambda_{\max }(U U)+2 \tau_{12}$ $\lambda_{\text {max }}\left(M_{1 i}\right) \lambda_{\text {max }}(U)+(1 / 2)\left[\tau_{21}^{3} \lambda_{\max }\left(R_{1 i}\right)+\left(\tau_{22}-\tau_{21}\right)^{3}\right.$ $\left.\left.\lambda_{\text {max }}\left(R_{2 i}\right)+\tau_{11}^{3} \lambda_{\max }\left(R_{3 i}\right)+\left(\tau_{12}-\tau_{11}\right)^{3} \lambda_{\max }\left(R_{4 i}\right)\right]\right\}, \lambda_{2}=$ $\min _{i \in \mathrm{N}}\left[\min \left(\lambda_{\min }\left(P_{1 i}\right), \lambda_{\min }\left(P_{2 i}\right)\right)\right]$. The equilibrium point of (6) is finite time stable with respect to positive real numbers $\left(c_{1}, c_{2}, T\right)$.

Proof. Based on the system (6), we construct the following Lyapunov-krasovskii functional:

$$
V_{\sigma(t)}(t)=V_{1 \sigma(t)}(t)+V_{2 \sigma(t)}(t)+V_{3 \sigma(t)}(t)
$$

where

$$
\begin{gathered}
V_{1 \sigma(t)}(t)=x^{T}(t) P_{1 \sigma(t)} x(t)+y^{T}(t) P_{2 \sigma(t)} y(t) \\
V_{2 \sigma(t)}(t)=\int_{t-\tau_{2}(t)}^{t} x^{T}(s) Q_{1 \sigma(t)} x(t) d s \\
+\int_{t-\tau_{1}(t)}^{t}\left[y^{T}(s), g^{T}(y(s))\right]\left[\begin{array}{ll}
Q_{2 \sigma(t)} & M_{1 \sigma(t)} \\
M_{1 \sigma(t)} & Q_{3 \sigma(t)}
\end{array}\right] \\
\times\left[\begin{array}{c}
y(s) \\
g(y(s))
\end{array}\right] d s
\end{gathered}
$$




$$
\begin{aligned}
& V_{3 \sigma(t)}(t) \\
& =\int_{-\tau_{21}}^{0} \int_{t+\theta}^{t} \tau_{21} \dot{x}^{T}(s) R_{1 \sigma(t)} \dot{x}(s) d s d \theta \\
& \quad+\int_{-\tau_{22}}^{-\tau_{21}} \int_{t+\theta}^{t}\left(\tau_{22}-\tau_{21}\right) \dot{x}^{T}(s) R_{2 \sigma(t)} \dot{x}(s) d s d \theta \\
& +\int_{-\tau_{11}}^{0} \int_{t+\theta}^{t} \tau_{11} \dot{y}^{T}(s) R_{3 \sigma(t)} \dot{y}(s) d s d \theta \\
& +\int_{-\tau_{12}}^{-\tau_{11}} \int_{t+\theta}^{t}\left(\tau_{12}-\tau_{11}\right) \dot{y}^{T}(s) R_{4 \sigma(t)} \dot{y}(s) d s d \theta .
\end{aligned}
$$

First, when $t \in\left[t_{k}, t_{k+1}\right)$, taking the derivatives of $V_{i}, i=1,2,3$ along the trajectory of system (6), we have that

$$
\begin{aligned}
& \dot{V}_{1 \sigma\left(t_{k}\right)}(t)=2 x^{T}(t) P_{1 \sigma\left(t_{k}\right)} \dot{x}(t)+2 y^{T}(t) P_{2 \sigma\left(t_{k}\right)} \dot{y}(t), \\
& \dot{V}_{2 \sigma\left(t_{k}\right)}(t)=x^{T}(t) Q_{1 \sigma\left(t_{k}\right)} x(t)-x^{T}\left(t-\tau_{2}(t)\right) Q_{1 \sigma\left(t_{k}\right)} x^{T} \\
& \times\left(t-\tau_{2}(t)\right)\left(1-\dot{\tau}_{2}(t)\right) \\
& +\left[y^{T}(t), g^{T}(y(t))\right]\left[\begin{array}{cc}
Q_{2 \sigma\left(t_{k}\right)} & M_{1 \sigma\left(t_{k}\right)} \\
* & Q_{3 \sigma\left(t_{k}\right)}
\end{array}\right] \\
& \times\left[\begin{array}{c}
y(t) \\
g(y(t))
\end{array}\right] \\
& -\left[y^{T}\left(t-\tau_{1}(t)\right), g^{T}\left(y\left(t-\tau_{1}(t)\right)\right)\right] \\
& \times\left[\begin{array}{cc}
Q_{2 \sigma\left(t_{k}\right)} & M_{1 \sigma\left(t_{k}\right)} \\
* & Q_{3 \sigma\left(t_{k}\right)}
\end{array}\right]\left[\begin{array}{c}
y\left(t-\tau_{1}(t)\right) \\
g\left(y\left(t-\tau_{1}(t)\right)\right)
\end{array}\right] \\
& \times\left(1-\dot{\tau}_{1}(t)\right), \\
& \dot{V}_{3 \sigma\left(t_{k}\right)}(t)=\tau_{21}^{2} \dot{x}^{T}(t) R_{1 \sigma\left(t_{k}\right)} \dot{x}(s) \\
& -\int_{t-\tau_{21}}^{t} \tau_{21} \dot{x}^{T}(\theta) R_{1 \sigma\left(t_{k}\right)} \dot{x}(\theta) d \theta \\
& +\left(\tau_{22}-\tau_{21}\right)^{2} \dot{x}^{T}(t) R_{2 \sigma\left(t_{k}\right)} \dot{x}(s) \\
& -\int_{t-\tau_{22}}^{t-\tau_{21}}\left(\tau_{22}-\tau_{21}\right) \dot{x}^{T}(\theta) R_{2 \sigma\left(t_{k}\right)} \dot{x}(\theta) d \theta \\
& +\tau_{11}^{2} \dot{y}^{T}(t) R_{3 \sigma\left(t_{k}\right)} \dot{y}(s) \\
& -\int_{t-\tau_{11}}^{t} \tau_{11} \dot{y}^{T}(\theta) R_{3 \sigma\left(t_{k}\right)} \dot{y}(\theta) d \theta \\
& +\left(\tau_{12}-\tau_{11}\right)^{2} \dot{y}^{T}(t) R_{4 \sigma\left(t_{k}\right)} \dot{y}(s) \\
& -\int_{t-\tau_{12}}^{t-\tau_{11}}\left(\tau_{12}-\tau_{11}\right) \dot{y}^{T}(\theta) R_{4 \sigma\left(t_{k}\right)} \dot{y}(\theta) d \theta .
\end{aligned}
$$

By Lemma 7, we have

$$
\begin{aligned}
& -\int_{t-\tau_{21}}^{t} \tau_{21} \dot{x}^{T}(\theta) R_{1 \sigma\left(t_{k}\right)} \dot{x}(\theta) d \theta \\
& \quad \leq-\left[x(t)-x\left(t-\tau_{21}\right)\right]^{T} R_{1 \sigma\left(t_{k}\right)}\left[x(t)-x\left(t-\tau_{21}\right)\right] \\
& -\int_{t-\tau_{11}}^{t} \tau_{11} \dot{y}^{T}(\theta) R_{3 \sigma\left(t_{k}\right)} \dot{y}(\theta) d \theta \\
& \quad \leq-\left[y(t)-y\left(t-\tau_{11}\right)\right]^{T} R_{3 \sigma\left(t_{k}\right)}\left[y(t)-y\left(t-\tau_{11}\right)\right]
\end{aligned}
$$

$-\int_{t-\tau_{22}}^{t-\tau_{21}}\left(\tau_{22}-\tau_{21}\right) \dot{x}^{T}(\theta) R_{2 \sigma\left(t_{k}\right)} \dot{x}(\theta) d \theta$ $=-\left(\tau_{22}-\tau_{21}\right)\left[\int_{t-\tau_{22}}^{t-\tau_{2}(t)} \dot{x}^{T}(\theta) R_{2 \sigma\left(t_{k}\right)} \dot{x}(\theta) d \theta\right.$ $\left.+\int_{t-\tau_{2}(t)}^{t-\tau_{21}} \dot{x}^{T}(\theta) R_{2 \sigma\left(t_{k}\right)} \dot{x}(\theta) d \theta\right]$ $=-\int_{t-\tau_{22}}^{t-\tau_{2}(t)}\left(\tau_{22}-\tau_{2}(t)\right) \dot{x}^{T}(\theta) R_{2 \sigma\left(t_{k}\right)} \dot{x}(\theta) d \theta$ $-\int_{t-\tau_{2}(t)}^{t-\tau_{21}}\left(\tau_{2}(t)-\tau_{21}\right) \dot{x}^{T}(\theta) R_{2 \sigma\left(t_{k}\right)} \dot{x}(\theta) d \theta$ $-\int_{t-\tau_{22}}^{t-\tau_{2}(t)}\left(\tau_{2}(t)-\tau_{21}\right) \dot{x}^{T}(\theta) R_{2 \sigma\left(t_{k}\right)} \dot{x}(\theta) d \theta$ $-\int_{t-\tau_{2}(t)}^{t-\tau_{21}}\left(\tau_{22}-\tau_{2}(t)\right) \dot{x}^{T}(\theta) R_{2 \sigma\left(t_{k}\right)} \dot{x}(\theta) d \theta$ $\leq-\left[x\left(t-\tau_{2}(t)\right)-x\left(t-\tau_{22}\right)\right]^{T}$ $\times R_{2 \sigma\left(t_{k}\right)}\left[x\left(t-\tau_{2}(t)\right)-x\left(t-\tau_{22}\right)\right]$ $-\left[x\left(t-\tau_{21}\right)-x\left(t-\tau_{2}(t)\right)\right]^{T}$ $\times R_{2 \sigma\left(t_{k}\right)}\left[x\left(t-\tau_{21}\right)-x\left(t-\tau_{2}(t)\right)\right]$ $-\frac{\tau_{2}(t)-\tau_{21}}{\tau_{22}-\tau_{21}}\left[x\left(t-\tau_{2}(t)\right)-x\left(t-\tau_{22}\right)\right]^{T}$ $\times R_{2 \sigma\left(t_{k}\right)}\left[x\left(t-\tau_{2}(t)\right)-x\left(t-\tau_{22}\right)\right]$ $-\frac{\tau_{22}-\tau_{2}(t)}{\tau_{22}-\tau_{21}}\left[x\left(t-\tau_{21}\right)-x\left(t-\tau_{2}(t)\right)\right]^{T}$ $\times R_{2 \sigma\left(t_{k}\right)}\left[x\left(t-\tau_{21}\right)-x\left(t-\tau_{2}(t)\right)\right]$. 
Similar to (20), we can obtain

$$
\begin{aligned}
&-\int_{t-\tau_{12}}^{t-\tau_{11}}\left(\tau_{12}-\tau_{11}\right) \dot{y}^{T}(\theta) R_{4 \sigma\left(t_{k}\right)} \dot{y}(\theta) d \theta \\
& \leq- {\left[y\left(t-\tau_{1}(t)\right)-y\left(t-\tau_{12}\right)\right]^{T} } \\
& \times R_{4 \sigma\left(t_{k}\right)}\left[y\left(t-\tau_{1}(t)\right)-y\left(t-\tau_{12}\right)\right] \\
&- {\left[y\left(t-\tau_{11}\right)-y\left(t-\tau_{1}(t)\right)\right]^{T} } \\
& \times R_{4 \sigma\left(t_{k}\right)}\left[y\left(t-\tau_{11}\right)-y\left(t-\tau_{1}(t)\right)\right] \\
&- \frac{\tau_{1}(t)-\tau_{11}}{\tau_{12}-\tau_{11}}\left[y\left(t-\tau_{1}(t)\right)-y\left(t-\tau_{12}\right)\right]^{T} \\
& \times R_{4 \sigma\left(t_{k}\right)}\left[y\left(t-\tau_{1}(t)\right)-y\left(t-\tau_{12}\right)\right] \\
&-\frac{\tau_{12}-\tau_{1}(t)}{\tau_{12}-\tau_{11}}\left[y\left(t-\tau_{11}\right)-y\left(t-\tau_{1}(t)\right)\right]^{T} \\
& \times R_{4 \sigma\left(t_{k}\right)}\left[y\left(t-\tau_{11}\right)-y\left(t-\tau_{1}(t)\right)\right] .
\end{aligned}
$$

In addition, for any $\Lambda_{m}=\operatorname{diag}\left(\lambda_{m 1}, \ldots, \lambda_{m n}\right) \geq 0, m=1,2$, the following inequality is true from (5):

$$
\begin{aligned}
& -2 \sum_{i=1}^{n} \lambda_{1 i} g_{i}\left(y_{i}(t)\right)\left[g_{i}\left(y_{i}(t)\right)-u_{i} y_{i}(t)\right] \\
& \quad-2 \sum_{i=1}^{n} \lambda_{2 i} g_{i}\left(y_{i}\left(t-\tau_{1}(t)\right)\right) \\
& \quad \times\left[g_{i}\left(y_{i}\left(t-\tau_{1}(t)\right)\right)-u_{i} y_{i}\left(t-\tau_{1}(t)\right)\right] \geq 0 .
\end{aligned}
$$

It can be written as matrix form:

$$
\begin{gathered}
-2 g^{T}(y(t)) \Lambda_{1}[g(y(t))-U y(t)]-2 g^{T}\left(y\left(t-\tau_{1}(t)\right)\right) \Lambda_{2} \\
\times\left[g\left(y\left(t-\tau_{1}(t)\right)\right)-U y\left(t-\tau_{1}(t)\right)\right] \geq 0 .
\end{gathered}
$$

From (15) to (23), we have that

$$
\begin{aligned}
& \dot{V}_{\sigma\left(t_{k}\right)}(t) \\
& \leq 2 x^{T}(t) P_{1 \sigma\left(t_{k}\right)}\left[-A_{\sigma\left(t_{k}\right)} x(t)+B_{\sigma\left(t_{k}\right)} g\left(y\left(t-\tau_{1}(t)\right)\right)\right] \\
& \quad+2 y^{T}(t) P_{2 \sigma\left(t_{k}\right)}\left[-C_{\sigma\left(t_{k}\right)} y(t)+D_{\sigma\left(t_{k}\right)} x\left(t-\tau_{2}(t)\right)\right] \\
& \quad+x^{T}(t) Q_{1 \sigma\left(t_{k}\right)} x(t)-x^{T}\left(t-\tau_{2}(t)\right) Q_{1 \sigma\left(t_{k}\right)} x^{T} \\
& \quad \times\left(t-\tau_{2}(t)\right)\left(1-\tau_{2 d}\right)+y^{T}(t) Q_{2 \sigma} y(t) \\
& \quad+2 y^{T}(t) M_{1 \sigma} g(y(t))+g^{T}(y(t)) Q_{3 \sigma\left(t_{k}\right)} g(y(t)) \\
& \quad-y^{T}\left(t-\tau_{1}(t)\right) Q_{2 \sigma\left(t_{k}\right)} y\left(t-\tau_{1}(t)\right)\left(1-\tau_{1 d}\right) \\
& \quad-2 y^{T}\left(t-\tau_{1}(t)\right) M_{1 \sigma\left(t_{k}\right)} g\left(y\left(t-\tau_{1}(t)\right)\right)\left(1-\tau_{1 d}\right)
\end{aligned}
$$

$$
\begin{aligned}
& -g^{T}\left(y\left(t-\tau_{1}(t)\right)\right) Q_{3 \sigma\left(t_{k}\right)} g\left(y\left(t-\tau_{1}(t)\right)\right)\left(1-\tau_{1 d}\right) \\
& +\tau_{21}^{2}\left[-A_{\sigma\left(t_{k}\right)} x(t)+B_{\sigma\left(t_{k}\right)} g\left(y\left(t-\tau_{1}(t)\right)\right)\right]^{T} \\
& \times R_{1 \sigma\left(t_{k}\right)}\left[-A_{\sigma\left(t_{k}\right)} x(t)+B_{\sigma\left(t_{k}\right)} g\left(y\left(t-\tau_{1}(t)\right)\right)\right] \\
& +\left(\tau_{22}-\tau_{21}\right)^{2}\left[-A_{\sigma\left(t_{k}\right)} x(t)+B_{\sigma\left(t_{k}\right)} g\left(y\left(t-\tau_{1}(t)\right)\right)\right]^{T} \\
& \times R_{2 \sigma\left(t_{k}\right)}\left[-A_{\sigma\left(t_{k}\right)} x(t)+B_{\sigma\left(t_{k}\right)} g\left(y\left(t-\tau_{1}(t)\right)\right)\right] \\
& +\tau_{11}^{2}\left[-C_{\sigma\left(t_{k}\right)} y(t)+D_{\sigma\left(t_{k}\right)} x\left(t-\tau_{2}(t)\right)\right]^{T} \\
& \times R_{3 \sigma\left(t_{k}\right)}\left[-C_{\sigma\left(t_{k}\right)} y(t)+D_{\sigma\left(t_{k}\right)} x\left(t-\tau_{2}(t)\right)\right] \\
& +\left(\tau_{12}-\tau_{11}\right)^{2}\left[-C_{\sigma\left(t_{k}\right)} y(t)+D_{\sigma\left(t_{k}\right)} x\left(t-\tau_{2}(t)\right)\right]^{T} \\
& \times R_{4 \sigma\left(t_{k}\right)}\left[-C_{\sigma\left(t_{k}\right)} y(t)+D_{\sigma\left(t_{k}\right)} x\left(t-\tau_{2}(t)\right)\right] \\
& -\left[x(t)-x\left(t-\tau_{21}\right)\right]^{T} R_{1 \sigma\left(t_{k}\right)}\left[x(t)-x\left(t-\tau_{21}\right)\right] \\
& -\left[y(t)-y\left(t-\tau_{11}\right)\right]^{T} R_{3 \sigma\left(t_{k}\right)}\left[y(t)-y\left(t-\tau_{11}\right)\right] \\
& -\left[x\left(t-\tau_{2}(t)\right)-x\left(t-\tau_{22}\right)\right]^{T} \\
& \times R_{2 \sigma\left(t_{k}\right)}\left[x\left(t-\tau_{2}(t)\right)-x\left(t-\tau_{22}\right)\right] \\
& -\left[x\left(t-\tau_{21}\right)-x\left(t-\tau_{2}(t)\right)\right]^{T} \\
& \times R_{2 \sigma\left(t_{k}\right)}\left[x\left(t-\tau_{21}\right)-x\left(t-\tau_{2}(t)\right)\right] \\
& -\left[y\left(t-\tau_{1}(t)\right)-y\left(t-\tau_{12}\right)\right]^{T} \\
& \times R_{4 \sigma\left(t_{k}\right)}\left[y\left(t-\tau_{1}(t)\right)-y\left(t-\tau_{12}\right)\right] \\
& -\left[y\left(t-\tau_{11}\right)-y\left(t-\tau_{1}(t)\right)\right]^{T} \\
& \times R_{4 \sigma\left(t_{k}\right)}\left[y\left(t-\tau_{11}\right)-y\left(t-\tau_{1}(t)\right)\right] \\
& -2 g^{T}(y(t)) \Lambda_{1}[g(y(t))-U y(t)] \\
& -2 g^{T}\left(y\left(t-\tau_{1}(t)\right)\right) \\
& \times \Lambda_{2}\left[g\left(y\left(t-\tau_{1}(t)\right)\right)-U y\left(t-\tau_{1}(t)\right)\right] \\
& \leq \xi^{T} \Xi \xi+\alpha\left(x^{T}(t) P_{1 \sigma\left(t_{k}\right)} x(t)+y^{T}(t) P_{2 \sigma\left(t_{k}\right)} y(t)\right) \\
& \leq \xi^{T} \Xi \xi+\alpha V_{\sigma\left(t_{k}\right)}(t),
\end{aligned}
$$

where

$$
\begin{aligned}
\xi^{T}=[ & x^{T}(t), x^{T}\left(t-\tau_{21}\right), x^{T}\left(t-\tau_{2}(t)\right), x^{T}\left(t-\tau_{22}\right), \\
& y^{T}(t), y^{T}\left(t-\tau_{11}\right), y^{T}\left(t-\tau_{1}(t)\right), y^{T}\left(t-\tau_{12}\right) \\
& \left.g^{T}(y(t)), g^{T}\left(y\left(t-\tau_{1}(t)\right)\right)\right] .
\end{aligned}
$$


By condition (13), we have

$$
\dot{V}_{\sigma\left(t_{k}\right)}(t) \leq \alpha V_{\sigma\left(t_{k}\right)}(t) \text {. }
$$

Integrating (26) from $t_{k}$ to $t$, we obtain that

$$
V_{\sigma\left(t_{k}\right)}(t) \leq e^{\alpha\left(t-t_{k}\right)} V_{\sigma\left(t_{k}\right)}\left(t_{k}\right)
$$

Using (12), at switching instant $t_{k}$, we have

$$
V_{\sigma\left(t_{k}\right)}\left(t_{k}\right) \leq \mu V_{\sigma\left(t_{k}^{-}\right)}\left(t_{k}^{-}\right)
$$

Therefore, it follows from (27) and (28) that

$$
V_{\sigma\left(t_{k}\right)}(t) \leq e^{\alpha\left(t-t_{k}\right)} \mu V_{\sigma\left(t_{k}^{-}\right)}\left(t_{k}^{-}\right) .
$$

For any $t \in[0, T]$, noting that $N_{\sigma}(0, t) \leq N_{\sigma}(0, T) \leq T / \tau_{a}$, we have

$$
V_{\sigma(t)}(t) \leq e^{\alpha t} \mu^{T / \tau_{a}} V_{\sigma(0)}(0)
$$

On the other hand, it follows from (15) that

$$
\begin{aligned}
& V_{\sigma(0)}(0) \\
& \leq \max _{i \in \mathbf{N}}\left\{\left[\lambda_{\max }\left(P_{1 i}\right)+\tau_{22} \lambda_{\max }\left(Q_{1 i}\right)\right]\right. \\
& \times \sup _{-\rho \leq t \leq 0}\|\varphi(t)\|^{2}+\left[\lambda_{\max }\left(P_{2 i}\right)+\tau_{12} \lambda_{\max }\left(Q_{2 i}\right)\right. \\
& +\tau_{12} \lambda_{\max }\left(Q_{3 i}\right) \lambda_{\max }(U U) \\
& \left.+2 \tau_{12} \lambda_{\max }\left(M_{1 i}\right) \lambda_{\max }(U)\right] \\
& \times \sup _{-\rho \leq t \leq 0}\|\psi(t)\|^{2}+\frac{1}{2}\left[\tau_{21}^{3} \lambda_{\max }\left(R_{1 i}\right)+\left(\tau_{22}-\tau_{21}\right)^{3}\right. \\
& \left.\times \lambda_{\max }\left(R_{2 i}\right)\right] \\
& \times \sup _{-\rho \leq t \leq 0}\|\dot{\varphi}(t)\|^{2}+\frac{1}{2}\left[\tau_{11}^{3} \lambda_{\max }\left(R_{3 i}\right)+\left(\tau_{12}-\tau_{11}\right)^{3}\right. \\
& \left.\left.\times \lambda_{\max }\left(R_{4 i}\right)\right] \sup _{-\rho \leq t \leq 0}\|\dot{\psi}(t)\|^{2}\right\}
\end{aligned}
$$

$$
\begin{aligned}
\leq \max _{i \in \mathbf{N}}\{ & \lambda_{\max }\left(P_{1 i}\right)+\tau_{22} \lambda_{\max }\left(Q_{1 i}\right)+\lambda_{\max }\left(P_{2 i}\right) \\
& +\tau_{12} \lambda_{\max }\left(Q_{2 i}\right)+\tau_{12} \lambda_{\max }\left(Q_{3 i}\right) \lambda_{\max }(U U) \\
& +2 \tau_{12} \lambda_{\max }\left(M_{1 i}\right) \lambda_{\max }(U) \\
& +\frac{1}{2}\left[\tau_{21}^{3} \lambda_{\max }\left(R_{1 i}\right)+\left(\tau_{22}-\tau_{21}\right)^{3} \lambda_{\max }\left(R_{2 i}\right)\right. \\
& \left.\left.+\tau_{11}^{3} \lambda_{\max }\left(R_{3 i}\right)+\left(\tau_{12}-\tau_{11}\right)^{3} \lambda_{\max }\left(R_{4 i}\right)\right]\right\} c_{1},
\end{aligned}
$$$$
V_{\sigma(t)}(t)
$$

$$
\begin{aligned}
& \geq \min _{i \in \mathbf{N}}\left[\lambda_{\min }\left(P_{1 i}\right)\|x(t)\|^{2}+\lambda_{\min }\left(P_{2 i}\right)\|y(t)\|^{2}\right] \\
& \geq \min _{i \in \mathbf{N}}\left[\min \left(\lambda_{\min }\left(P_{1 i}\right), \lambda_{\min }\left(P_{2 i}\right)\right)\right] \\
& \quad \times\left(\|x(t)\|^{2}+\|y(t)\|^{2}\right) .
\end{aligned}
$$

From (30) and (31) it is easy to obtain

$$
\|x(t)\|^{2}+\|y(t)\|^{2} \leq e^{\alpha t} \mu^{T / \tau_{a}} \frac{\lambda_{1}}{\lambda_{2}} c_{1} \leq e^{\alpha T} \mu^{T / \tau_{a}} \frac{\lambda_{1}}{\lambda_{2}} c_{1},
$$

where

$$
\begin{aligned}
& \lambda_{1}=\max _{i \in \mathbf{N}}\{ \lambda_{\max }\left(P_{1 i}\right)+\tau_{22} \lambda_{\max }\left(Q_{1 i}\right)+\lambda_{\max }\left(P_{2 i}\right) \\
&+\tau_{12} \lambda_{\max }\left(Q_{2 i}\right)+\tau_{12} \lambda_{\max }\left(Q_{3 i}\right) \lambda_{\max }(U U) \\
&+2 \tau_{12} \lambda_{\max }\left(M_{1 i}\right) \lambda_{\max }(U) \\
&+\frac{1}{2}\left[\tau_{21}^{3} \lambda_{\max }\left(R_{1 i}\right)+\left(\tau_{22}-\tau_{21}\right)^{3} \lambda_{\max }\left(R_{2 i}\right)\right. \\
&\left.\left.\quad+\tau_{11}^{3} \lambda_{\max }\left(R_{3 i}\right)+\left(\tau_{12}-\tau_{11}\right)^{3} \lambda_{\max }\left(R_{4 i}\right)\right]\right\}, \\
& \lambda_{2}=\min _{i \in \mathbf{N}}\left[\min \left(\lambda_{\min }\left(P_{1 i}\right), \lambda_{\min }\left(P_{2 i}\right)\right)\right]
\end{aligned}
$$

By (14) and (32), for any $t \in[0, T]$, we obtain that

$$
\|x(t)\|^{2}+\|y(t)\|^{2} \leq c_{2} .
$$

This completes the proof.

Next, we consider the finite-time stability of the genetic regulatory networks with time-varying delays and unbounded continuous distributed delays:

$$
\begin{gathered}
\dot{x}(t)=-A_{\sigma(t)} x(t)+B_{\sigma(t)} g\left(y\left(t-\tau_{1}(t)\right)\right) \\
+W_{\sigma(t)} \int_{0}^{\infty} h(s) g(y(t-s)) d s, \\
\dot{y}(t)=-C_{\sigma(t)} y(t)+D_{\sigma(t)} x\left(t-\tau_{2}(t)\right),
\end{gathered}
$$

where $\sigma(t):[0, \infty) \rightarrow \mathbf{N}=\{1, \ldots, N\}$ is the switching signal, the matrix $W_{i}, i \in \mathbf{N}$, is the $i$ th subsystem distributively 
delayed connection weight matrix of appropriate dimension, $h(\cdot)=\operatorname{diag}\left(h_{1}(\cdot), \ldots, h_{n}(\cdot)\right)$ is the delay kernel function, and all the other signs are defined as (6).

Assumption 9. The delay kernel $h_{j}$ 's are some real value nonnegative continuous functions defined in $[0, \infty)$, that satisfy

$$
\begin{array}{r}
\int_{0}^{\infty} h_{j}(s) d s=1, \quad \int_{0}^{\infty} s h_{j}(s) d s=k_{i}<\infty, \\
j=1, \ldots, n .
\end{array}
$$

Theorem 10. For switching system (35) with Assumptions 1-9, given a scalar $\alpha>0$, if there exist symmetric positive definite matrices $P_{1 i}, P_{2 i}, Q_{1 i}, R_{1 i}, R_{2 i}, R_{3 i}, R_{4 i}$, the positive definite diagonal matrix $L_{i}=\operatorname{diag}\left(l_{1 i}, \ldots, l_{n i}\right)$, for all $i \in \mathbf{N}, \Lambda_{m}=$ $\operatorname{diag}\left(\lambda_{m 1}, \ldots, \lambda_{m n}\right), m=1,2$, matrices $Q_{2 i}, Q_{3 i}, M_{1 i}, i \in N$, and positive scalars $\mu \geq 1$ such that the following inequalities

$$
\begin{gathered}
{\left[\begin{array}{cc}
Q_{2 i} & M_{1 i} \\
* & Q_{3 i}
\end{array}\right] \geq 0, \quad P_{k i} \leq \mu P_{k j}, \quad k=1,2,} \\
{\left[\begin{array}{cc}
Q_{2 i} & M_{1 i} \\
* & Q_{3 i}
\end{array}\right] \leq \mu\left[\begin{array}{cc}
Q_{2 j} & M_{1 j} \\
* & Q_{3 j}
\end{array}\right], \quad R_{l i} \leq \mu R_{l j},} \\
l=1, \ldots, 4, \quad \forall i, j \in \mathbf{N},
\end{gathered}
$$

$$
\Xi_{1}=\Omega_{1}
$$

$$
\begin{aligned}
+\operatorname{sym}\{ & \left\{F_{1}^{T} R_{1 i} F_{2}+F_{1}^{T}\left[P_{1 i} B_{i}-\tau_{21}^{2} B_{i}^{T} R_{1 i} A_{i}\right.\right. \\
& \left.-\left(\tau_{22}-\tau_{21}\right)^{2} B_{i}^{T} R_{2 i} A_{i}\right] \\
& \times F_{10}+F_{2}^{T} R_{2 i} F_{3}+F_{3}^{T} R_{2 i} F_{4}+F_{3}^{T} \\
& \times\left[P_{2 i} D_{i}-\tau_{11}^{2} D_{i}^{T} R_{3 i} C_{i}-\left(\tau_{12}-\tau_{11}\right)^{2} D_{i}^{T} R_{4 i} C_{i}\right] \\
& \times F_{5}+F_{5}^{T} R_{3 i} F_{6}+F_{5}^{T}\left(M_{1 i}+\Lambda_{1} U\right) F_{9}+F_{6}^{T} R_{4 i} F_{7} \\
& \left.+F_{7}^{T} R_{4 i} F_{8}+F_{7}^{T}\left[M_{1 i}\left(1-\tau_{13}\right)+\Lambda_{2} U\right] F_{10}\right\}
\end{aligned}
$$

$<0, \quad \forall i \in \mathbf{N}$,

hold, and the average dwell time of the switch signal $\sigma(t)$ satisfies

$$
\tau_{a}>\tau_{a}^{*}=\frac{T \ln \mu}{\ln c_{2} \lambda_{2}-\ln c_{1} \lambda_{1}-\alpha T},
$$

where $\Omega=\operatorname{diag}\left(-2 P_{1 i} A_{i}+\tau_{21}^{2} A_{i}^{T} R_{1 i} A_{i}+\left(\tau_{22}-\tau_{21}\right)^{2}\right.$ $A_{i}^{T} R_{2 i} A_{i}-R_{1 i}-\alpha P_{1 i},-R_{1 i}-R_{2 i},-Q_{1 i}\left(1-\tau_{23}\right)+\tau_{11}^{2} D_{i}^{T}$ $R_{3 i} D_{i}+\left(\tau_{12}-\tau_{11}\right)^{2} D_{i}^{T} R_{4 i} D_{i}-2 R_{2 i},-R_{2 i},-2 P_{2 i} C_{i}+Q_{2 i}+\tau_{11}^{2} C_{i}^{T}$ $R_{3 i} C_{i}+\left(\tau_{12}-\tau_{11}\right)^{2} C_{i}^{T} R_{4 i} C_{i}-R_{3 i}-\alpha P_{2 i},-R_{3 i}-R_{4 i},-Q_{2 i}$ $\left(1-\tau_{13}\right)-2 R_{4 i},-R_{4 i}, Q_{2 i}-2 \Lambda_{1}+L_{i},-Q_{3 i}\left(1-\tau_{13}\right)+$ $\left.\tau_{21}^{2} B_{i}^{T} R_{1 i} B_{i}+\left(\tau_{22}-\tau_{21}\right)^{2} B_{i}^{T} R_{2 i} B_{i}-2 \Lambda_{2},-L_{i}\right), F_{j}=\left[0_{n \times(j-1) n}\right.$, $\left.I_{n \times n}, 0_{n \times(12-j) n}\right], j=1, \ldots, 11, \lambda_{1}^{\prime}=\max _{i \in \mathbf{N}}\left\{\lambda_{\max }\left(P_{1 i}\right)+\right.$ $\tau_{22} \lambda_{\max }\left(Q_{1 i}\right)+\lambda_{\max }\left(P_{2 i}\right)+\tau_{12} \lambda_{\max }\left(Q_{2 i}\right)+$ $\tau_{12} \lambda_{\text {max }}\left(Q_{3 i}\right) \lambda_{\text {max }}(U U)+2 \tau_{12} \lambda_{\max }\left(M_{1 i}\right) \lambda_{\text {max }}(U)+(1 / 2)$ $\left[\tau_{21}^{3} \lambda_{\text {max }}\left(R_{1 i}\right)+\left(\tau_{22}-\tau_{21}\right)^{3} \lambda_{\text {max }}\left(R_{2 i}\right)+\tau_{11}^{3} \lambda_{\text {max }}\left(R_{3 i}\right)+\right.$ $\left.\left.\left(\tau_{12}-\tau_{11}\right)^{3} \lambda_{\text {max }}\left(R_{4 i}\right)\right]+\lambda_{\text {max }}\left(R_{4 i}\right)+G^{2} \sum_{j=1}^{n} l_{i j} k_{j}\right\}, \lambda_{2}^{\prime}=\lambda_{2}$. The equilibrium point of (35) is finite time stable with respect to positive real numbers $\left(c_{1}, c_{2}, T\right)$.

Proof. Based on the system (35), we construct the following Lyapunov-krasovskii functional:

$$
V_{\sigma(t)}(t)=V_{1 \sigma(t)}(t)+V_{2 \sigma(t)}(t)+V_{3 \sigma(t)}(t)+V_{4 \sigma(t)}(t),
$$

where $V_{1 \sigma(t)}(t), V_{2 \sigma(t)}(t), V_{3 \sigma(t)}(t)$ are defined as in Theorem 8 ,

$$
V_{4 \sigma(t)}(t)=\sum_{i=1}^{n} l_{i \sigma(t)} \int_{0}^{\infty} h_{i}(\theta) \int_{t-\theta}^{t} g_{i}^{2}\left(y_{i}(s)\right) d s d \theta,
$$

when $t \in\left[t_{k}, t_{k+1}\right)$; taking the derivatives of $V_{4}$ along the trajectory of system (35), we have that

$$
\begin{aligned}
\dot{V}_{4 \sigma\left(t_{k}\right)}(t)= & \sum_{i=1}^{n} l_{i \sigma\left(t_{k}\right)} g_{i}^{2}\left(y_{i}(t)\right) \\
& -\sum_{i=1}^{n} l_{i \sigma\left(t_{k}\right)} \int_{0}^{\infty} h_{i}(\theta) g_{i}^{2}\left(y_{i}(t-\theta)\right) d \theta,
\end{aligned}
$$

By Cauchy's inequality $\left(\int p(s) q(s) d s\right)^{2} \leq\left(\int p^{2}(s) d s\right)$ $\left(\int q^{2}(s) d s\right)$, we obtain that

$$
\begin{aligned}
\sum_{i=1}^{n} l_{i \sigma\left(t_{k}\right)} & \int_{0}^{\infty} h_{i}(\theta) g_{i}^{2}\left(y_{i}(t-\theta)\right) d \theta \\
= & \sum_{i=1}^{n} l_{i \sigma\left(t_{k}\right)} \int_{0}^{\infty} h_{i}(\theta) d \theta \\
& \times \int_{0}^{\infty} h_{i}(\theta) g_{i}^{2}\left(y_{i}(t-\theta)\right) d \theta \\
\geq & \sum_{i=1}^{n} l_{i \sigma\left(t_{k}\right)}\left[\int_{0}^{\infty} h_{i}(\theta) g_{i}\left(y_{i}(t-\theta)\right) d \theta\right]^{2} \\
= & {\left[\int_{0}^{\infty} h_{i}(\theta) g_{i}\left(y_{i}(t-\theta)\right) d \theta\right]^{T} } \\
& \times L_{\sigma\left(t_{k}\right)}\left[\int_{0}^{\infty} h_{i}(\theta) g_{i}\left(y_{i}(t-\theta)\right) d \theta\right] .
\end{aligned}
$$

Therefore,

$$
\begin{aligned}
\dot{V}_{4 \sigma\left(t_{k}\right)}(t) \leq & g^{T}(y(t)) L_{\sigma\left(t_{k}\right)} g(y(t)) \\
& -\left[\int_{0}^{\infty} h_{i}(\theta) g_{i}\left(y_{i}(t-\theta)\right) d \theta\right]^{T} \\
& \times L_{\sigma\left(t_{k}\right)}\left[\int_{0}^{\infty} h_{i}(\theta) g_{i}\left(y_{i}(t-\theta)\right) d \theta\right] .
\end{aligned}
$$

Combing (17)-(23) and (40)-(43), we get

$$
\dot{V}_{\sigma\left(t_{k}\right)}(t) \leq \xi_{1}^{T} \Xi_{1} \xi_{1}+\alpha V_{\sigma\left(t_{k}\right)}(t),
$$


where

$$
\begin{aligned}
\xi_{1}^{T}=\left[x^{T}(t), x^{T}\left(t-\tau_{21}\right), x^{T}\left(t-\tau_{2}(t)\right), x^{T}\left(t-\tau_{22}\right),\right. \\
y^{T}(t), y^{T}\left(t-\tau_{11}\right), y^{T}\left(t-\tau_{1}(t)\right), y^{T}\left(t-\tau_{12}\right), \\
g^{T}(y(t)), g^{T}\left(y\left(t-\tau_{1}(t)\right)\right), \\
\left.\quad\left[\int_{0}^{\infty} h_{i}(\theta) g_{i}\left(y_{i}(t-\theta)\right) d \theta\right]^{T}\right] .
\end{aligned}
$$

Similar to the proof of Theorem 8 , for any $t \in[0, T]$, we have that

$$
\|x(t)\|^{2}+\|y(t)\|^{2} \leq e^{\alpha T} \mu^{T / \tau_{a}} \frac{\lambda_{1}^{\prime}}{\lambda_{2}^{\prime}} c_{1},
$$

where

$$
\begin{aligned}
& \lambda_{1}^{\prime}=\max _{i \in \mathbf{N}}\{ \lambda_{\max }\left(P_{1 i}\right)+\tau_{22} \lambda_{\max }\left(Q_{1 i}\right)+\lambda_{\max }\left(P_{2 i}\right) \\
&+\tau_{12} \lambda_{\max }\left(Q_{2 i}\right)+\tau_{12} \lambda_{\max }\left(Q_{3 i}\right) \lambda_{\max }(U U) \\
&+2 \tau_{12} \lambda_{\max }\left(M_{1 i}\right) \lambda_{\max }(U) \\
&+\frac{1}{2}\left[\tau_{21}^{3} \lambda_{\max }\left(R_{1 i}\right)+\left(\tau_{22}-\tau_{21}\right)^{3} \lambda_{\text {max }}\left(R_{2 i}\right)\right. \\
&\left.+\tau_{11}^{3} \lambda_{\max }\left(R_{3 i}\right)+\left(\tau_{12}-\tau_{11}\right)^{3} \lambda_{\max }\left(R_{4 i}\right)\right] \\
&\left.+\lambda_{\max }\left(R_{4 i}\right)+G^{2} \sum_{j=1}^{n} l_{i j} k_{j}\right\}, \\
& \lambda_{2}^{\prime}=\lambda_{2} .
\end{aligned}
$$

By (39) and (47), we obtain that

$$
\|x(t)\|^{2}+\|y(t)\|^{2} \leq c_{2} .
$$

This completes the proof.

\section{Numerical Examples}

In this section, we will give two examples to show the effectiveness of our results. The aim is to examine the finitetime stability for gene networks under proper conditions by applying Theorems 8 and 10 .

Example 1. Consider a genetic regulatory network model reported by Elowitz and Leibler [27], which studied the dynamics of repressilator which is cyclic negative-feedback

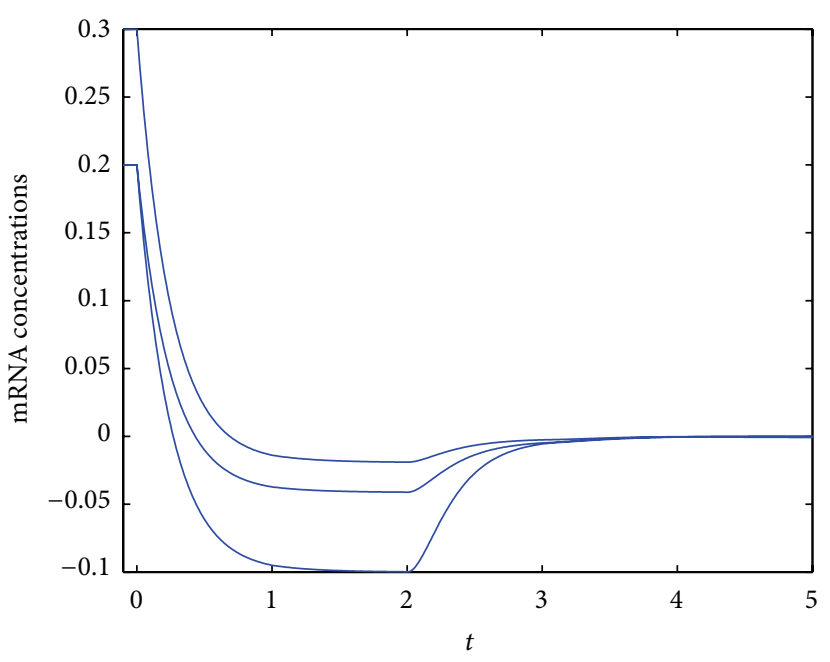

FIGURE 1: mRNA concentrations $x(t)$.

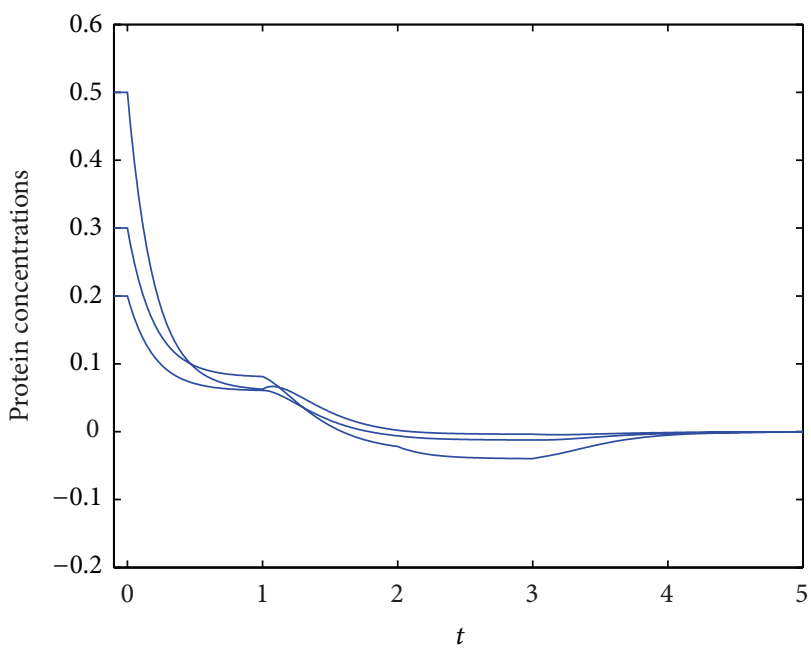

FIgURE 2: Protein concentrations $y(t)$.

loop comprising three repressor genes $(i=l a c l$, tet $R$, and $c l)$ and their promoters $(j=c l$, lacl, and tet $R)$ :

$$
\begin{gathered}
\frac{d m_{i}}{d t}=-m_{i}+\frac{\alpha}{1+p_{j}^{n}}+\alpha_{0}, \\
\frac{d p_{i}}{d t}=\beta\left(m_{i}-p_{i}\right),
\end{gathered}
$$

where $m_{i}$ and $p_{i}$ are the concentrations of two mRNAs and repressor-protein; $\alpha_{0}$ is the growth rate of protein in a cell in the presence of saturating amounts of repressor, while $\alpha_{0}+\alpha$ is the growth rate in its abscence; $\beta$ denotes the ratio of the protein decay rate to mRNA decay rate; and $n$ is a Hill coefficient.

Taking time-varying delays and mode switching into account, we rewrite the above equation into vector form by 
adjusting some parameters and shifting the equilibrium point to the origin, and then we get the following model:

$$
\begin{gathered}
\dot{x}(t)=-A_{i} x(t)+B_{i} g(y(t-\sigma(t))), \\
\dot{y}(t)=-C_{i} y(t)+D_{i} x(t-\tau(t)), \quad i=1,2,
\end{gathered}
$$

where

$$
\begin{aligned}
& A_{1}=\operatorname{diag}(4,4,4), \quad C_{1}=\operatorname{diag}(5,5,5), \\
& D_{1}=\operatorname{diag}(2,1.5,1), \quad A_{2}=\operatorname{diag}(3,3,3) \text {, } \\
& C_{2}=\operatorname{diag}(4,4,4), \quad D_{2}=\operatorname{diag}(1.2,1.2,1.2) \text {, } \\
& B_{1}=2 \times\left(\begin{array}{ccc}
0 & 0 & -1 \\
-1 & 0 & 0 \\
0 & -1 & 0
\end{array}\right), \quad B_{2}=1.5 \times\left(\begin{array}{ccc}
0 & 0 & -1 \\
-1 & 0 & 0 \\
0 & -1 & 0
\end{array}\right) \text {. }
\end{aligned}
$$

$$
\Xi=\left(\begin{array}{cccccccccc}
a_{11}^{i} & R_{1 i} & 0 & 0 & 0 & 0 & 0 & 0 & 0 & a_{1,10}^{i} \\
* & -R_{1 i}-R_{2 i} & R_{2 i} & 0 & 0 & 0 & 0 & 0 & 0 & 0 \\
* & * & a_{33}^{i} & R_{2 i} & a_{3,5}^{i} & 0 & 0 & 0 & 0 & 0 \\
* & * & * & -R_{2 i} & 0 & 0 & 0 & 0 & 0 & 0 \\
* & * & * & * & a_{55}^{i} & R_{3 i} & 0 & 0 & M_{1 i}+\Lambda_{1} U & 0 \\
* & * & * & * & * & -R_{3 i}-R_{4 i} & R_{4 i} & 0 & 0 & 0 \\
* & * & * & * & * & * & a_{77}^{i} & R_{4 i} & 0 & a_{7,10}^{i} \\
* & * & * & * & * & * & * & -R_{4 i} & 0 & 0 \\
* & * & * & * & * & * & * & * & Q_{3 i}-2 \Lambda_{1} & 0 \\
* & * & * & * & * & * & * & * & * & a_{10,10}^{i}
\end{array}\right),
$$

where $a_{11}^{i}=-2 P_{1 i} A_{i}+Q_{1 i}+\tau_{21}^{2} A_{i}^{T} R_{1 i} A_{i}+\left(\tau_{22}-\right.$ $\left.\tau_{21}\right)^{2} A_{i}^{T} R_{2 i} A_{i}-R_{1 i}-\alpha P_{1 i}, a_{1,10}^{i}=P_{1 i} B_{i}-\tau_{21}^{2} B_{i}^{T} R_{1 i} A_{i}-$ $\left(\tau_{22}-\tau_{21}\right)^{2} B_{i}^{T} R_{2 i} A_{i}, a_{33}^{i}=-Q_{1 i}\left(1-\tau_{23}\right)+\tau_{11}^{2} D_{i}^{T} R_{3 i} D_{i}+$ $\left(\tau_{12}-\tau_{11}\right)^{2} D_{i}^{T} R_{4 i} D_{i}-2 R_{2 i}, a_{35}^{i}=P_{2 i} D_{i}-\tau_{11}^{2} D_{i}^{T} R_{3 i} C_{i}-\left(\tau_{12}-\right.$ $\left.\tau_{11}\right)^{2} D_{i}^{T} R_{4 i} C_{i}, a_{55}^{i}=-2 P_{2 i} C_{i}+Q_{2 i}+\tau_{11}^{2} C_{i}^{T} R_{3 i} C_{i}+\left(\tau_{12}-\right.$ $\left.\tau_{11}\right)^{2} C_{i}^{T} R_{4 i} C_{i}-R_{3 i}-\alpha P_{2 i}, a_{77}^{i}=-Q_{2 i}\left(1-\tau_{13}\right)-2 R_{4 i}, a_{7,10}^{i}=$ $M_{1 i}\left(1-\tau_{13}\right)+\Lambda_{2} U, a_{10,10}^{i}=-Q_{3 i}\left(1-\tau_{13}\right)+\tau_{21}^{2} B_{i}^{T} R_{1 i} B_{i}+$ $\left(\tau_{22}-\tau_{21}\right)^{2} B_{i}^{T} R_{2 i} B_{i}-2 \Lambda_{2}, i=1,2$.

Due to the space limitation, we only list matrices $P_{11}, P_{12}$, $Q_{11}$, and $Q_{12}$ here:

$$
\begin{aligned}
& P_{11}=\left(\begin{array}{ccc}
3.5732 & -0.2655 & -0.3039 \\
-0.2655 & 3.3575 & -0.2912 \\
-0.3039 & -0.2912 & 3.1531
\end{array}\right), \\
& P_{12}=\left(\begin{array}{ccc}
4.6463 & -0.1506 & -0.1453 \\
-0.1506 & 4.7212 & -0.1477 \\
-0.1453 & -0.1477 & 4.7809
\end{array}\right), \\
& Q_{11}=\left(\begin{array}{ccc}
9.1959 & -0.3223 & -0.2832 \\
-0.3223 & 7.4980 & -0.2791 \\
-0.2832 & -0.2791 & 5.9270
\end{array}\right),
\end{aligned}
$$

The gene regulation function is taken as $g(x)=x^{2} /(1+$ $\left.x^{2}\right), U=\operatorname{diag}(0.65,0.65,0.65)$. The time delays $\tau_{1}(t)$ and $\tau_{2}(t)$ are assumed to be:

$$
\tau_{1}(t)=0.6+0.4 \sin t, \quad \tau_{2}(t)=0.3+0.1 \cos t ;
$$

we can get the parameters as following:

$$
\begin{array}{ccc}
\tau_{11}=0.2, & \tau_{12}=1, & \tau_{13}=0.4, \\
\tau_{21}=0.4, & \tau_{22}=0.2, & \tau_{23}=0.1,
\end{array}
$$

and let $c_{1}=2, c_{2}=1.5, T=5, \alpha=0.02, \mu=1.2$. By using the Matlab LMI toolbox, we can solve the LMIs (12), (13) and obtain feasible solutions. Equation (13) can be reformulated in the following in the form of LMI:

$$
Q_{12}=\left(\begin{array}{rrr}
10.8205 & -0.4573 & -0.4597 \\
-0.4573 & 10.9025 & -0.4104 \\
-0.4597 & -0.4104 & 11.0402
\end{array}\right)
$$

and $\tau_{a}>\tau_{a}^{*}=0.4003$. The initial condition is $x(0)=$ $(0.2,0.2,0.3)^{T}, y(0)=(0.3,0.2,0.5)^{T}$. The simulation results of the trajectories are shown in Figures 1 and 2.

Example 2. In this example, we consider the genetic regulatory (35) with time-varying delays and unbounded continuous distributed delays, in which the parameters are listed as follows:

$$
\begin{array}{cc}
A_{1}=\operatorname{diag}(2.1,1.2,2.5), & C_{1}=\operatorname{diag}(4.6,4,5.2), \\
D_{1}=\operatorname{diag}(0.5,0.4,0.6), & A_{2}=\operatorname{diag}(1.8,2,3.1), \\
C_{2}=\operatorname{diag}(5,4,5), & D_{2}=\operatorname{diag}(0.3,0.3,0.4), \\
B_{1}=\left(\begin{array}{lll}
0 & 1 & 0 \\
0 & 0 & 1 \\
1 & 0 & 0
\end{array}\right), & B_{2}=\left(\begin{array}{ccc}
0 & 0.7 & 0 \\
0 & 0 & 0.7 \\
0.7 & 0 & 0
\end{array}\right),
\end{array}
$$


and $g(x)=x^{2} /\left(1+x^{2}\right), U=\operatorname{diag}(0.65,0.65,0.65)$. The time delays $\tau_{1}(t)$ and $\tau_{2}(t)$ are assumed to be

$$
\tau_{1}(t)=0.5+0.3 \sin t, \quad \tau_{2}(t)=0.4+0.1 \cos t ;
$$

we can get the parameters as following:

$$
\begin{array}{lll}
\tau_{11}=0.2, & \tau_{12}=0.8, & \tau_{13}=0.3, \\
\tau_{21}=0.3, & \tau_{22}=0.5, & \tau_{23}=0.1,
\end{array}
$$

and let $c_{1}=2.5, c_{2}=2, T=5, \alpha=0.02, \mu=1.2, h_{j}(s)=e^{-s}$, and $G=1$. By using the Matlab LMI toolbox, we can solve the LMIs (37) and (38) and obtain feasible solutions. Equation (38) can be reformulated in the following in the form of LMI:

$$
\Xi=\left(\begin{array}{ccccccccccc}
a_{11}^{i} & R_{1 i} & 0 & 0 & 0 & 0 & 0 & 0 & 0 & a_{0}^{i} & 0 \\
* & -R_{1 i}-R_{2 i} & R_{2 i} & 0 & 0 & 0 & 0 & 0 & 0 & 0 & 0 \\
* & * & a_{33}^{i} & R_{2 i} & a_{3,5}^{i} & 0 & 0 & 0 & 0 & 0 & 0 \\
* & * & * & -R_{2 i} & 0 & 0 & 0 & 0 & 0 & 0 & 0 \\
* & * & * & * & a_{55}^{i} & R_{3 i} & 0 & 0 & M_{1 i}+\Lambda_{1} U & 0 & 0 \\
* & * & * & * & * & -R_{3 i}-R_{4 i} & R_{4 i} & 0 & 0 & 0 & 0 \\
* & * & * & * & * & * & a_{77}^{i} & R_{4 i} & 0 & a_{7,10}^{i} & 0 \\
* & * & * & * & * & * & * & -R_{4 i} & 0 & 0 & 0 \\
* & * & * & * & * & * & * & * & a_{99}^{i} & 0 & 0 \\
* & * & * & * & * & * & * & * & * & a_{10,10}^{i} & 0 \\
* & * & * & * & * & * & * & * & * & * & -L_{i}
\end{array}\right),
$$

where $a_{11}^{i}=-2 P_{1 i} A_{i}+\tau_{21}^{2} A_{i}^{T} R_{1 i} A_{i}+\left(\tau_{22}-\tau_{21}\right)^{2} A_{i}^{T} R_{2 i} A_{i}-$ $R_{1 i}-\alpha P_{1 i}, a_{1,10}^{i}=P_{1 i} B_{i}-\tau_{21}^{2} B_{i}^{T} R_{1 i} A_{i}-\left(\tau_{22}-\tau_{21}\right)^{2} B_{i}^{T} R_{2 i} A_{i}$, $a_{33}^{i}=-Q_{1 i}\left(1-\tau_{23}\right)+\tau_{11}^{2} D_{i}^{T} R_{3 i} D_{i}+\left(\tau_{12}-\tau_{11}\right)^{2} D_{i}^{T} R_{4 i} D_{i}-$ $2 R_{2 i}, a_{35}^{i}=P_{2 i} D_{i}-\tau_{11}^{2} D_{i}^{T} R_{3 i} C_{i}-\left(\tau_{12}-\tau_{11}\right)^{2} D_{i}^{T} R_{4 i} C_{i}, a_{55}^{i}=$ $-2 P_{2 i} C_{i}+Q_{2 i}+\tau_{11}^{2} C_{i}^{T} R_{3 i} C_{i}+\left(\tau_{12}-\tau_{11}\right)^{2} C_{i}^{T} R_{4 i} C_{i}-R_{3 i}-\alpha P_{2 i}$, $a_{77}^{i}=-Q_{2 i}\left(1-\tau_{13}\right)-2 R_{4 i}, a_{7,10}^{i}=M_{1 i}\left(1-\tau_{13}\right)+\Lambda_{2} U, a_{99}^{i}=$ $Q_{2 i}-2 \Lambda_{1}+L_{i}, a_{10,10}^{i}=-Q_{3 i}\left(1-\tau_{13}\right)+\tau_{21}^{2} B_{i}^{T} R_{1 i} B_{i}+\left(\tau_{22}-\right.$ $\left.\tau_{21}\right)^{2} B_{i}^{T} R_{2 i} B_{i}-2 \Lambda_{2}, i=1,2$.

Due to the space limitation, we only list matrices $P_{11}, P_{12}$, $Q_{11}$, and $Q_{12}$ here:

$$
\begin{aligned}
P_{11} & =\left(\begin{array}{ccc}
1.3745 & -0.0657 & -0.0145 \\
-0.0657 & 1.3683 & -0.0471 \\
-0.0145 & -0.0471 & 1.2893
\end{array}\right), \\
P_{12} & =\left(\begin{array}{ccc}
1.6025 & -0.0233 & -0.0056 \\
-0.0233 & 1.5143 & -0.0073 \\
-0.0056 & -0.0073 & 1.2907
\end{array}\right), \\
Q_{11} & =\left(\begin{array}{ccc}
2.5617 & -0.0905 & -0.0313 \\
-0.0905 & 2.0794 & -0.0718 \\
-0.0313 & -0.0718 & 2.4995
\end{array}\right), \\
Q_{12} & =\left(\begin{array}{ccc}
2.1367 & -0.0751 & -0.0258 \\
-0.0751 & 2.1184 & -0.0434 \\
-0.0258 & -0.0434 & 2.2000
\end{array}\right),
\end{aligned}
$$

and $\tau_{a}>\tau_{a}^{*}=0.5012$.

\section{Concluding Remarks}

This paper has investigated the finite-time stability for switching genetic regulatory networks with interval timevarying delays and unbounded continuous distributed delays. By structuring appropriate Lyapunov-Krasovskii functionals method, several sufficient conditions of switching genetic networks were obtained, which guarantee that the system is finite-time stable. Finally, two numerical examples illustrated our results' validity.

\section{Conflict of Interests}

The author declares that there is no conflict of interests regarding the publication of this paper.

\section{Acknowledgments}

This work was supported by the National Natural Science Foundations of China (61273084, 61233014, and 61174217), the Natural Science Foundation for Distinguished Young Scholar of Shandong Province of China (JQ200919), the Independent Innovation Foundation of Shandong University (2012JC014), the Natural Science Foundation of Shandong Province of China (ZR2010AL016 and ZR2011AL007), and the Doctoral Foundation of University of Jinan (XBS1244).

\section{References}

[1] S. A. Kauffman, "Metabolic stability and epigenesis in randomly constructed genetic nets," Journal of Theoretical Biology, vol. 22, 
no. 3, pp. 437-467, 1969.

[2] X. Lou, Q. Ye, and B. Cui, "Exponential stability of genetic regulatory networks with random delays," Neurocomputing, vol. 73, no. 4-6, pp. 759-769, 2010.

[3] F.-X. wu, "Delay-independent stability of genetic regulatory networks," IEEE Transactions on Neural Network, vol. 22, no. 11, pp. 1685-1693, 2011.

[4] W. Pan, Z. Wang, H. Gao, and X. Liu, "Monostability and multistability of genetic regulatory networks with different types of regulation functions," Nonlinear Analysis: Real World Applications, vol. 11, no. 4, pp. 3170-3185, 2010.

[5] H. Li, "Asymptotic stability of stochastic genetic networks with disturbance attenuation," in Proceedings of the 30th Chinese Control Conference (CCC '11), pp. 5697-5702, Yantai, China, July 2011.

[6] W. Zhang, J.-A. Fang, and Y. Tang, "Robust stability for genetic regulatory networks with linear fractional uncertainties," Communications in Nonlinear Science and Numerical Simulation, vol. 17, no. 4, pp. 1753-1765, 2012.

[7] H. Li and X. Yang, "Asymptotic stability analysis of genetic regulatory networks with time-varying delay," in Proceedings of the Chinese Control and Decision Conference (CCDC '10), pp. 566-571, Xuzhou, China, May 2010.

[8] W. Zhang, J.-A. Fang, and Y. Tang, "New robust stability analysis for genetic regulatory networks with random discrete delays and distributed delays," Neurocomputing, vol. 74, no. 14-15, pp. 2344-2360, 2011.

[9] X. Lou, Q. Ye, and B. Cui, "Exponential stability of genetic regulatory networks with random delays," Neurocomputing, vol. 73, no. 4-6, pp. 759-769, 2010.

[10] W. Wang and S. Zhong, "Delay-dependent stability criteria for genetic regulatory networks with time-varying delays and nonlinear disturbance," Communications in Nonlinear Science and Numerical Simulation, vol. 17, no. 9, pp. 3597-3611, 2012.

[11] R. Rakkiyappan and P. Balasubramaniam, "Delay-probabilitydistribution-dependent stability of uncertain stochastic genetic regulatory networks with mixed time-varying delays: an LMI approach," Nonlinear Analysis: Hybrid Systems, vol. 4, no. 3, pp. 600-607, 2010.

[12] W. Wang and S. Zhong, "Stochastic stability analysis of uncertain genetic regulatory networks with mixed time-varying delays," Neurocomputing, vol. 82, pp. 143-156, 2012.

[13] J. Cao and F. Ren, "Exponential stability of discrete-time genetic regulatory networks with delays," IEEE Transactions on Neural Networks, vol. 19, no. 3, pp. 520-523, 2008.

[14] F.-X. Wu, "Stability analysis of genetic regulatory networks with multiple time delays," in Proceedings of the 29th Annual International Conference of the IEEE, Engineering in Medicine and Biology Society (EMBS '07), pp. 1387-1390, Lyon, France, Aughust 2007.

[15] Z. Wang, X. Liao, S. Guo, and H. Wu, "Mean square exponential stability of stochastic genetic regulatory networks with timevarying delays," Information Sciences, vol. 181, no. 4, pp. 792-811, 2011.

[16] S. Kim, H. Li, E. R. Dougherty et al., "Can Markov chain models mimic biological regulation?” Journal of Biological Systems, vol. 10, no. 4, pp. 337-357, 2002.

[17] X. Li, R. Rakkiyappan, and C. Pradeep, "Robust $\mu$-stability analysis of Markovian switching uncertain stochastic genetic regulatory networks with unbounded time-varying delays," Communications in Nonlinear Science and Numerical Simulation, vol. 17, no. 10, pp. 3894-3905, 2012.
[18] W. Zhang, J.-A. Fang, and Y. Tang, "Stochastic stability of Markovian jumping genetic regulatory networks with mixed time delays," Applied Mathematics and Computation, vol. 217, no. 17, pp. 7210-7225, 2011.

[19] D. Zhang and L. Yu, "Passivity analysis for stochastic Markovian switching genetic regulatory networks with time-varying delays," Communications in Nonlinear Science and Numerical Simulation, vol. 16, no. 8, pp. 2985-2992, 2011.

[20] Y. Yao, J. Liang, and J. Cao, "Stability analysis for switched genetic regulatory networks: an average dwell time approach," Journal of the Franklin Institute, vol. 348, no. 10, pp. 2718-2733, 2011.

[21] J. Cheng, S. Zhong, Q. Zhong, H. Zhu, and Y. Du, "Finite-time boundedness of state estimation for neural networks with timevarying delays," Neurocomputing, 2014.

[22] J. Cheng, H. Zhu, S. Zhong, Y. Zeng, and X. Dong, "Finite-time $H_{\infty}$ control for a class of Markovian jump systems with modedependent time-varying delays via new Lyapunov functionals," ISA Transactions, vol. 52, no. 6, pp. 768-774, 2013.

[23] J. Zhou, S. Xu, and H. Shen, "Finite-time robust stochastic stability of uncertain stochastic delayed reaction-diffusion genetic regulatory networks," Neurocomputing, vol. 74, no. 17, pp. 27902796, 2011.

[24] Y. Sun, G. Feng, and J. Cao, "Stochastic stability of Markovian switching genetic regulatory networks," Physics Letters A, vol. 373, no. 18-19, pp. 1646-1652, 2009.

[25] J. Liang, J. Lam, and Z. Wang, "State estimation for Markov-type genetic regulatory networks with delays and uncertain mode transition rates," Physics Letters, Section A, vol. 373, no. 47, pp. 4328-4337, 2009.

[26] W. Su and Y. Chen, "Global robust stability criteria of stochastic Cohen-Grossberg neural networks with discrete and distributed time-varying delays," Communications in Nonlinear Science and Numerical Simulation, vol. 14, no. 2, pp. 520-528, 2009.

[27] M. B. Elowitz and S. Leibler, "A synthetic oscillatory network of transcriptional regulators," Nature, vol. 403, no. 1, pp. 335-338, 2000. 


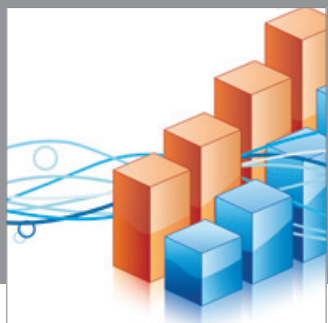

Advances in

Operations Research

mansans

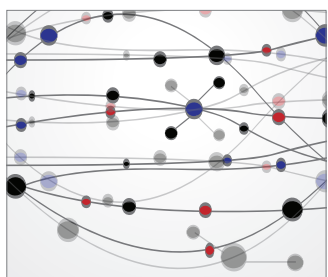

The Scientific World Journal
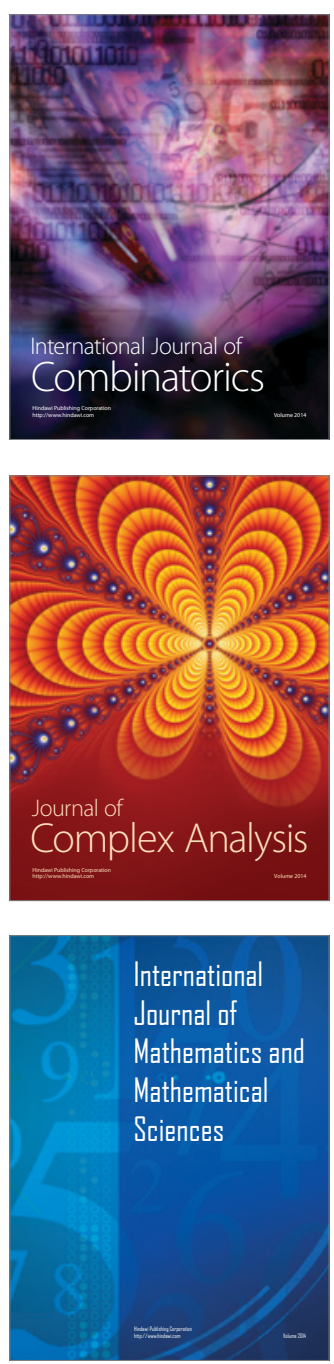
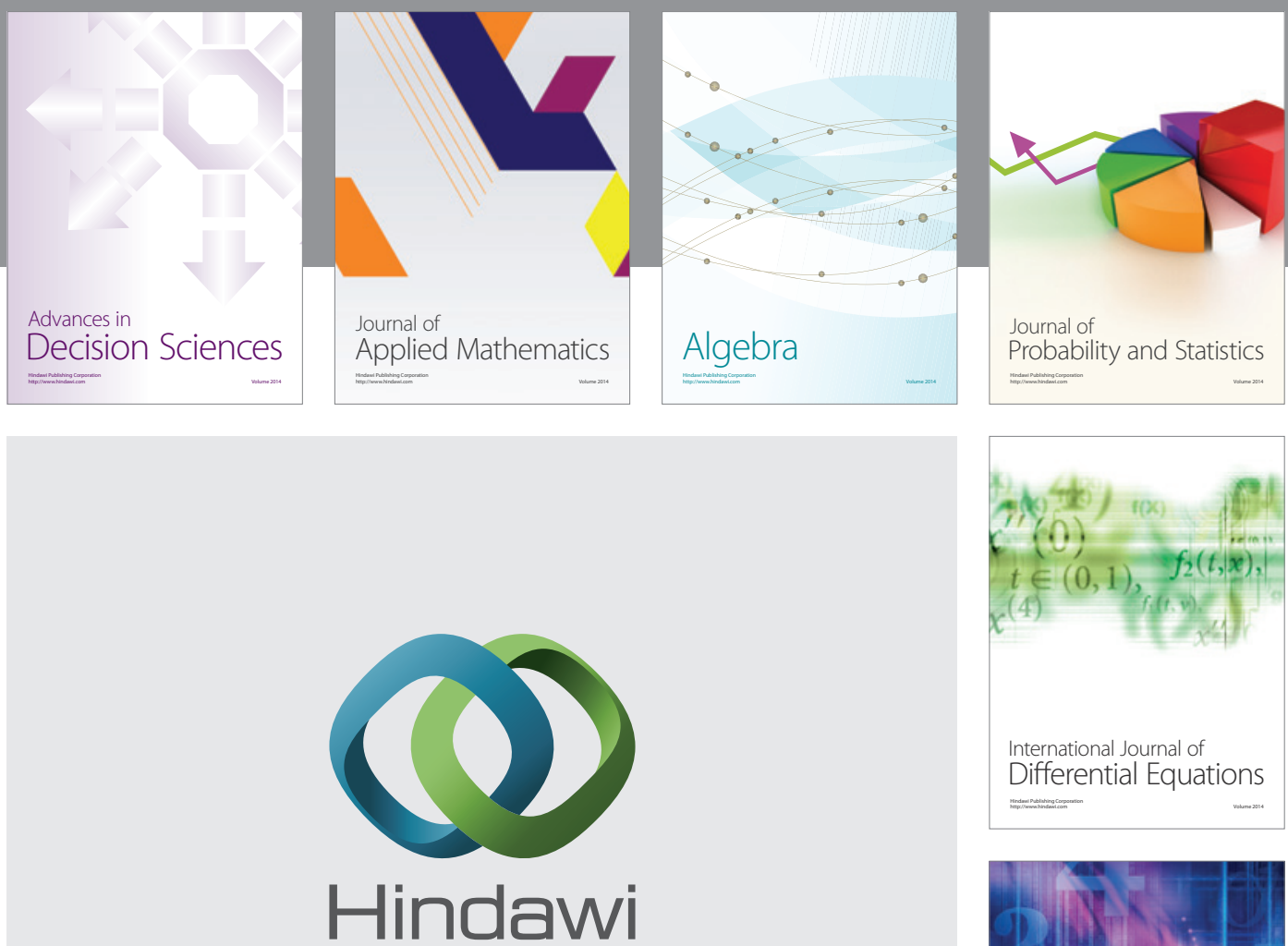

Submit your manuscripts at http://www.hindawi.com
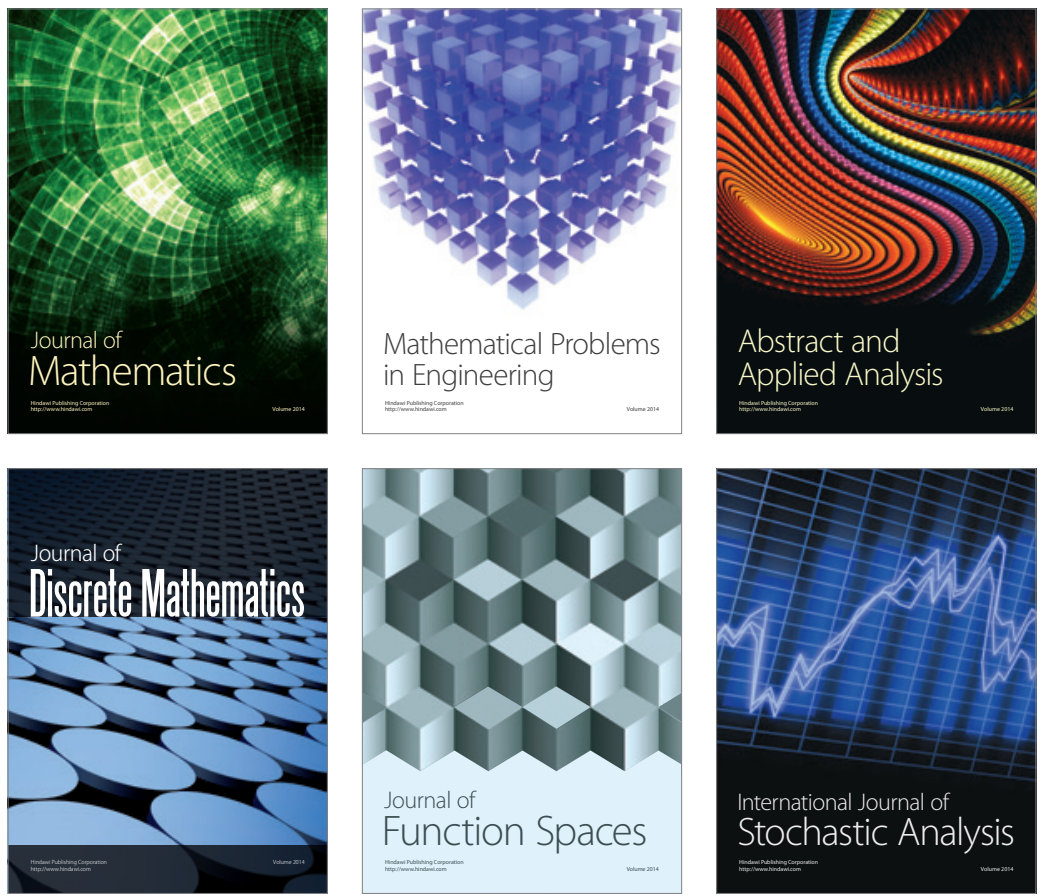

Journal of

Function Spaces

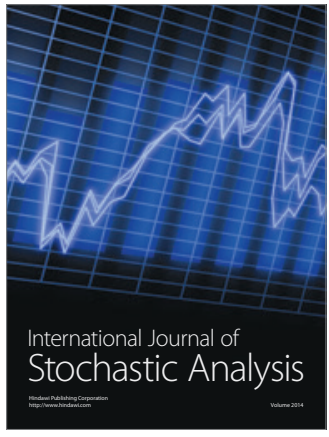

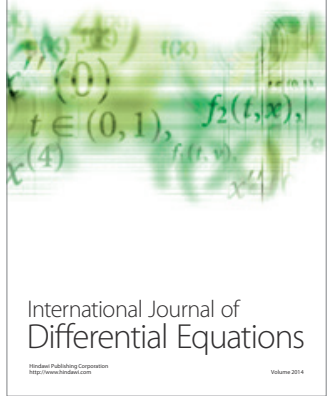
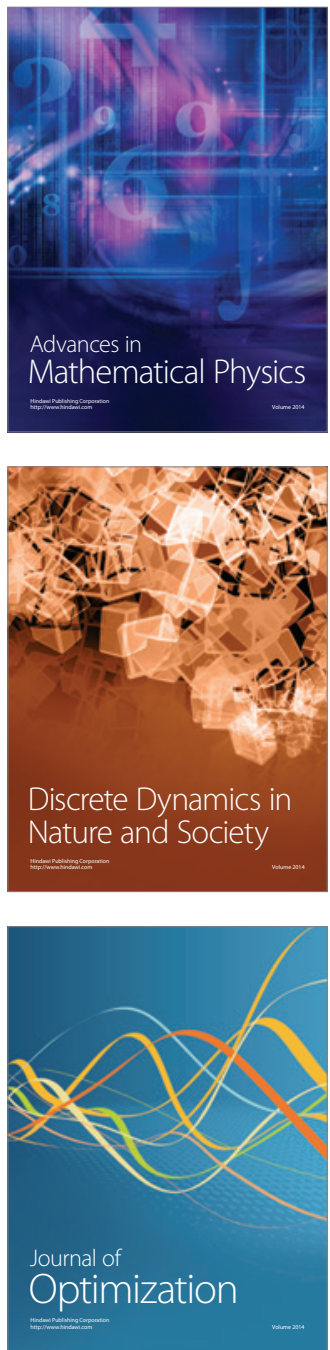